\title{
Comparison between OQPSK and DPSK Bidirectional Radio over Fiber Transmission Systems
}

\author{
Fady I. El-Nahal", Mamoun A. Salha \\ Department of Electrical Engineering, Islamic University of Gaza, Gaza, Gaza Strip , Palestinian Territories \\ *Corresponding Author: fnahal@iugaza.edu.ps
}

Copyright (C) 2013 Horizon Research Publishing All rights reserved.

\begin{abstract}
Radio over fiber is becoming an increasingly important technology for the wireless market since it introduces a higher data transmission rate and large bandwidth. In this paper, we have compared OQPSK and DPSK bidirectional radio over fiber ROF systems, where an offset quadrature phase shift keying (OQPSK) or differential phase shift keying (DPSK) signals are used for down-link and an on-off keying (OOK) signal re-modulated for up-link. Several measurements were performed including Bit Error Rate (BER) curves for uplink and downlink, RSOA gain curve and noise figure with the variation of input power and temperature for each system.
\end{abstract}

Keywords Radio Over Fiber (Rof), Reflective Semiconductor Optical Amplifier (RSOA), Offset Quadrature Phase Shift Keying (OQPSK), Differential Phase Shift Keying (DPSK)

\section{Introduction}

The proliferation of wireless devices coupled with increased demand for broadband services are putting pressure on wireless systems to increase capacity. So that wireless communications should enter a new phase to meet multimedia services [1]. To achieve this, wireless systems must have increased feeder network capacity, operate at higher carrier frequencies, and cope with increased user population densities. The raising of the carrier frequency would limit propagation characteristics; as a consequence, small cell sizes are formed. This leads to a large number of cells in a certain area, and then large number of remote antenna base stations (BSs) is required to cover an operational geographical area. These BSs provide wireless connectivity to users via millimeter-wave and are connected with a central office (CO) via an optical fiber access network. These leading to costly radio systems while the high installation and maintenance costs associated with high-bandwidth silica fiber render it economically impractical for in-home and office environments.
RoF technology is a technology by which microwave (electrical) signals are distributed by means of optical components and techniques. A RoF system consists of a Central Site (CS) and a Remote Site (RS) connected by an optical fiber link or network. If the application area is in a GSM network, then the CS could be the Mobile Switching Centre (MSC) and the RS is the base station (BS). For wireless Local Area Networks (WLANs), the CS would be the head end while the Radio Access Point (RAP) would act as the RS [2].

In several studies of bidirectional systems, a reflective semiconductor optical amplifier (RSOA) operating as a modulator often plays an important role, and both the upstream and down-stream channels use the same wavelength. This strategy is very useful for improving the wavelength utilization efficiency [3].

The functionality of RoF application technology exceeds modulation and frequency conversion to include signal processing such as filtering and attenuation control at high frequencies which referred to as microwave functions. Many of these functions are difficult to achieve in the electrical domain due to limited bandwidth and other electromagnetic wave propagation limitations. However, if the processing is done in the optical domain, unlimited signal processing bandwidth becomes available. As a result, many microwave functions can be performed by optical components without needing $\mathrm{E} / \mathrm{O}$ conversion for processing by microwave components and vice versa [4].

Radio-Over-Fiber (RoF) technology and Reflective Semiconductor Optical Amplifier (RSOA) provide a budget effective approach for reducing radio system costs because it simplifies the remote antenna sites since RSOA work as an amplifier and modulator, the project also enhances the sharing of expensive radio equipment located at Central Sites (CS). In this work, OQPSK and DPSK bidirectional radio over fiber ROF systems have been compared, where an offset quadrature phase shift keying (OQPSK) or differential phase shift keying (DPSK) signals are used for down-link and an on-off keying (OOK) signal re-modulated for up-link. The system was modeled using Optisystem software from Optiwave. 


\section{System Architecture}

The basic architecture used to model RoF is shown in Fig.1. Firstlly, we will study the OQPSK system. For down-link, a series of Continuous Wave (CW) lasers with various wavelengths are modulated by phase modulators using $1 \mathrm{~Gb} / \mathrm{s}$ non-return to zero (NRZ) downstream data to generate the desired downstream signal. The generated signal is sent over the bidirectional Single-Mode Fiber (SMF). A circulator is used in the central office (CO) to separate the downstream and upstream traffic. The modulated signals are sent to Optical Network Unit (ONU). At the ONU, using optical splitter/coupler, portion of the modulated signal is fed to a balanced receiver. For up-link, the other portion of the downstream modulated signal from the splitter/coupler is re-modulated using $1 \mathrm{~Gb} / \mathrm{s} \mathrm{NRZ}$ upstream data by RSOA in the ONU. The re-modulated OOK signals re-pass through the bidirectional SMF. By using the circulator to avoid influencing the downstream signals, the upstream signals are sent to a P-type Intrinsic N-type (PIN) receiver in the CO. The system model is categorized into three main parts which are Central Office (CO), single mode fiber channel, Remote Station (RS) or Base Station (BS).

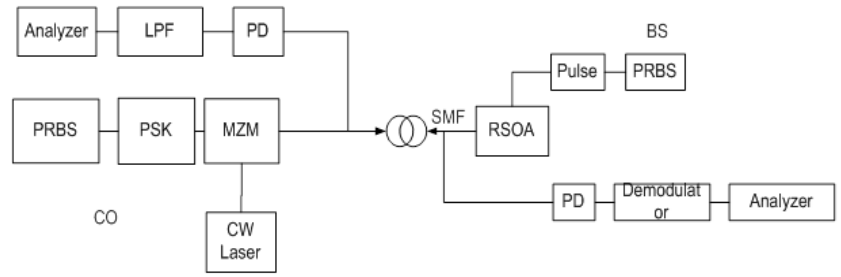

Figure 1. Schematic of a bidirectional RoF system.

The input data of the system is a pseudo random bit sequence at $1 \mathrm{G}$ bps bit rate and it is directly modulated using OQPSK modulator at a frequency equals $2 \mathrm{GHz}$ with normalized signal amplitude and $45^{\circ}$ phase offset with $0 \mathrm{DC}$ biasing. The OQPSK modulated signal is illustrated in Fig.2, the central frequency of the signal is $2 \mathrm{GHz}$ with $2 \mathrm{GHz}$ major bandwidth from $1 \mathrm{GHz}$ to $3 \mathrm{GHz}$ in two sidebands. The parameters of $\mathrm{CW}$ laser are configured as shown in Table 1.

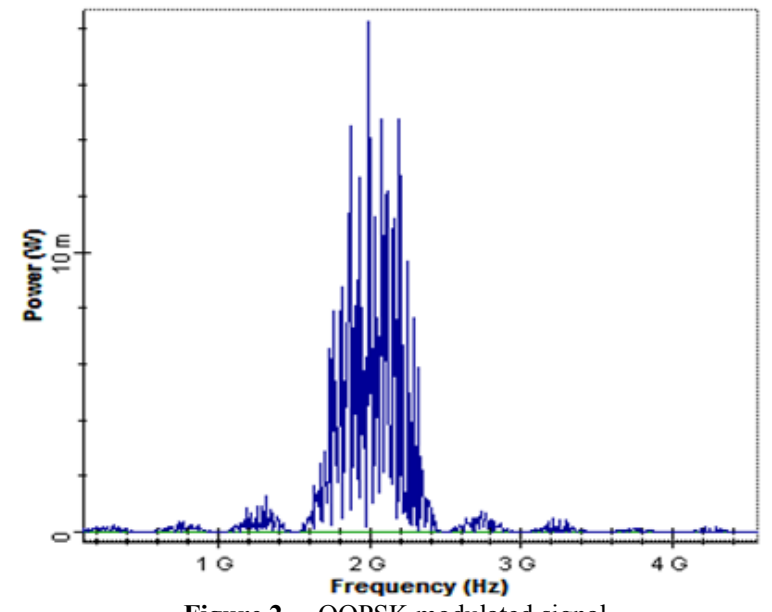

Figure 2. OQPSK modulated signal.
Table 1. The CW laser parameter

\begin{tabular}{|c|c|}
\hline Parameter & Value \\
\hline Frequency & $193.1 \mathrm{THz}$ \\
\hline Power & $(0-12) \mathrm{dBm}$ \\
\hline Line width & $10 \mathrm{MHz}$ \\
\hline Initial Phase & 0 degree \\
\hline
\end{tabular}

The Mach Zehnder Modulator has three ports: the first port is for electrical modulation type, the second is the $\mathrm{CW}$ laser input and the third port is the outlet of output optical signal. The extinction ratio is set to $30 \mathrm{~dB}$ to characterize the division power ratio of the upper path to lower path. The output optical signal is shown in Fig.3. It is clear from the figure that there is symmetry around $193.1 \mathrm{THz}$. The output power is measured by using the optical power meter before and after the circulator; $\mathrm{P}_{\text {out (Before) }}=8.99 \mathrm{dBm}$ and Pout ${ }_{\text {(After) }}$ $=7.99 \mathrm{dBm}$, the power loss is due to the insertion loss of the circulator.

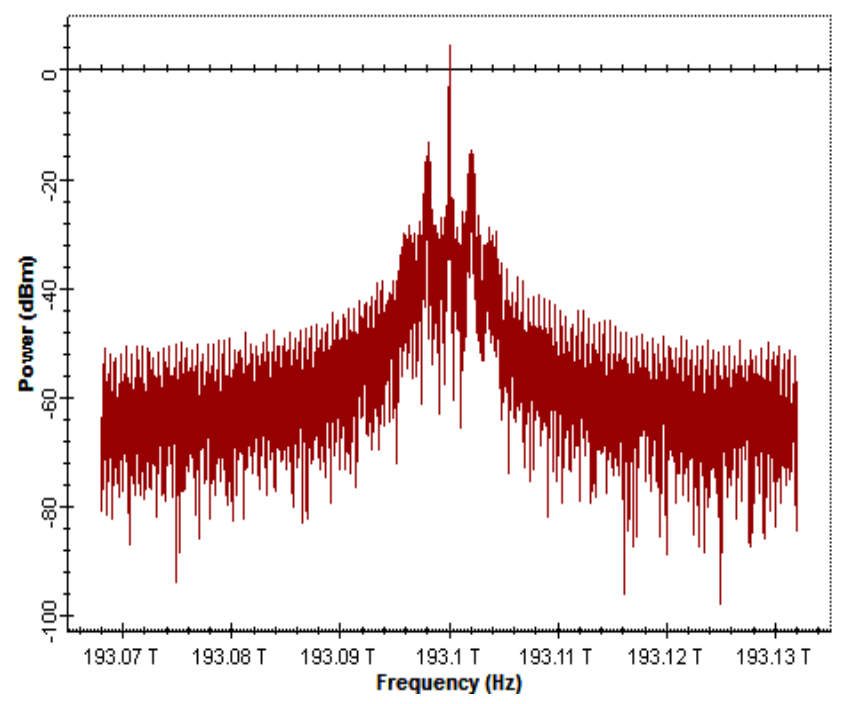

Figure 3. The MZM transmitted signal at $193.1 \mathrm{THz}$.

A single mode fiber of $50 \mathrm{~km}$ with an attenuation loss of $0.22 \mathrm{~dB} / \mathrm{km}$ is used; this means there is a $11 \mathrm{~dB}$ power loss. The signal passes the optical fiber to another circulator which also has $1 \mathrm{~dB}$ insertion loss and so the resultant power signal decreases $1 \mathrm{dBm}$ to become $-4 \mathrm{dBm}$, then the signal is distributed into two paths the first towards RSOA branch and the second towards downlink stage receiver. The optical signal is received by photodetector PD operating at 193.1 $\mathrm{THz}$ frequency to convert it back to electrical form, the received signal after photo detection is illustrated in Fig.4.

It is clear that the central frequency of the signal is about 2 $\mathrm{GHz}$ with major bandwidth equal to $12 \mathrm{GHz}$; also it is clear that there is a power loss and signal distortion due to conversion process, the signal power decreases to -42.775 $\mathrm{dBm}$. To recover the message signal; a band pass Bessel filter (BPF) is used with central frequency of $2 \mathrm{GHz}$ and 0.8 $\mathrm{GHz}$ bandwidth. 


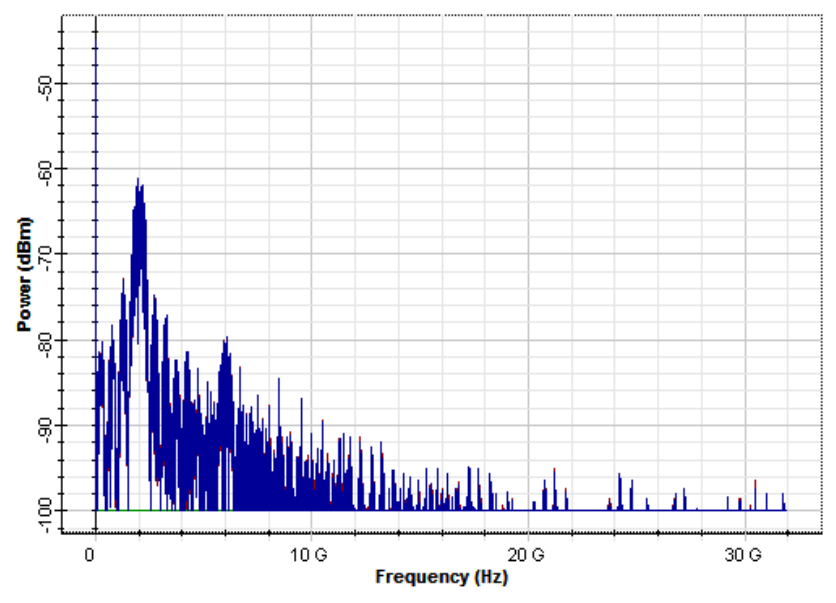

Figure 4. The electrical signal after PD.

The Reflective Semiconductor Optical Amplifier (RSOA) performs the functions of not only modulator but also amplifier. It is used for modulations conversion; it performs intensity modulations with no need for local laser source. In general, RSOA has six ports. Here; we use one port to merge the electrical pulse shaped signal at $1 \mathrm{G}$ bps for modulation purpose, another input port is used for optical transmitted OQPSK modulated signal, and the last output port to outlet the reflected optical intensity modulated signal to reinter the optical fiber cable in the opposite direction. A time delay is used to avoid collisions in a two optical directions. Fig.5 illustrates the output reflected signal from RSOA, the noise level in the graph which is due to phase to intensity modulation conversion inside RSOA [6,7].

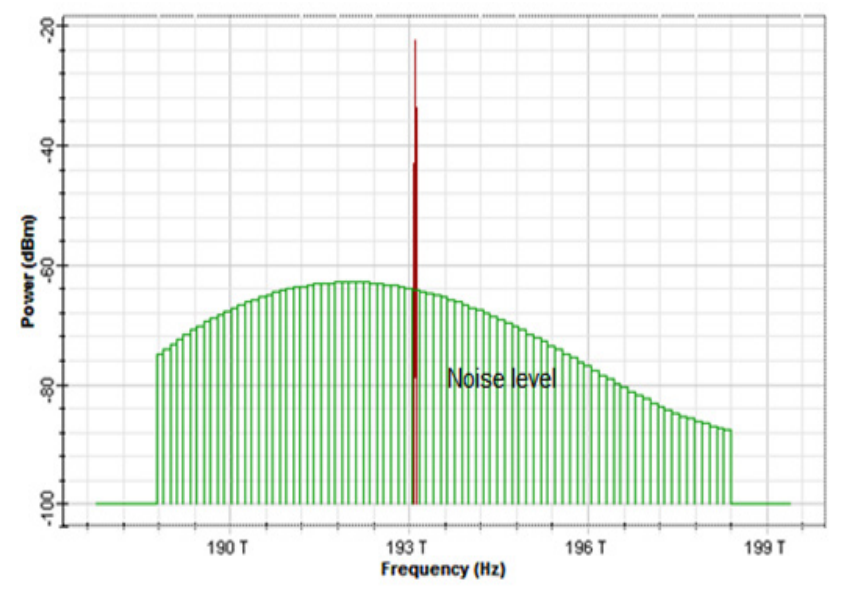

Figure 5. The reflected signal from RSOA.

The uplink receiver consists of a photodetector PD followed by a low pass filter LPF; the BER is displayed at the central office using BER analyzer.

\section{Results and Discussions}

\subsection{Offset Quadrature Phase Shift Keying (OQPSK)}

Our system consists of two main stages; the downlink stage and the uplink stage. Fig. 6 and Fig.7 illustrate the eye diagram of the downstream and upstream signal respectively for input power of $10 \mathrm{dBm}$. The received eye diagrams of downstream and upstream signals were measured at base station and central office respectively. In both downlink stage and uplink stage, we could see that the eye was clear and open. Though upstream was a thicker when it is compared with the downstream eye diagram. This could be attributed to the effect of phase to intensity conversion during RSOA injection. Table 2 compares between two stages, it is clear that the system performs more efficient in the downstream [8].

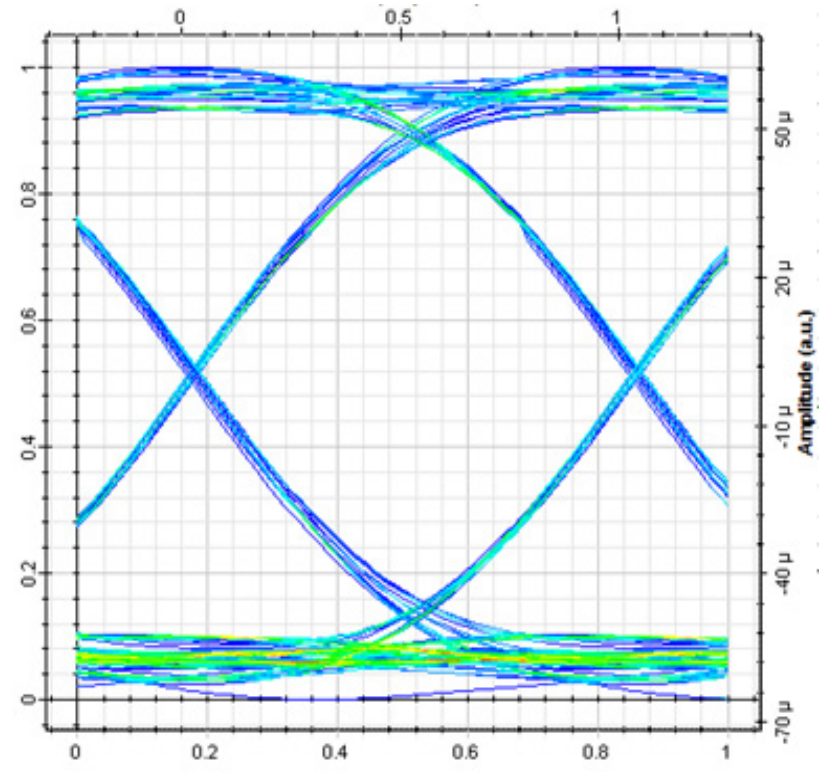

Figure 6. Eye diagram of the downstream signal at input power of $10 \mathrm{dBm}$.

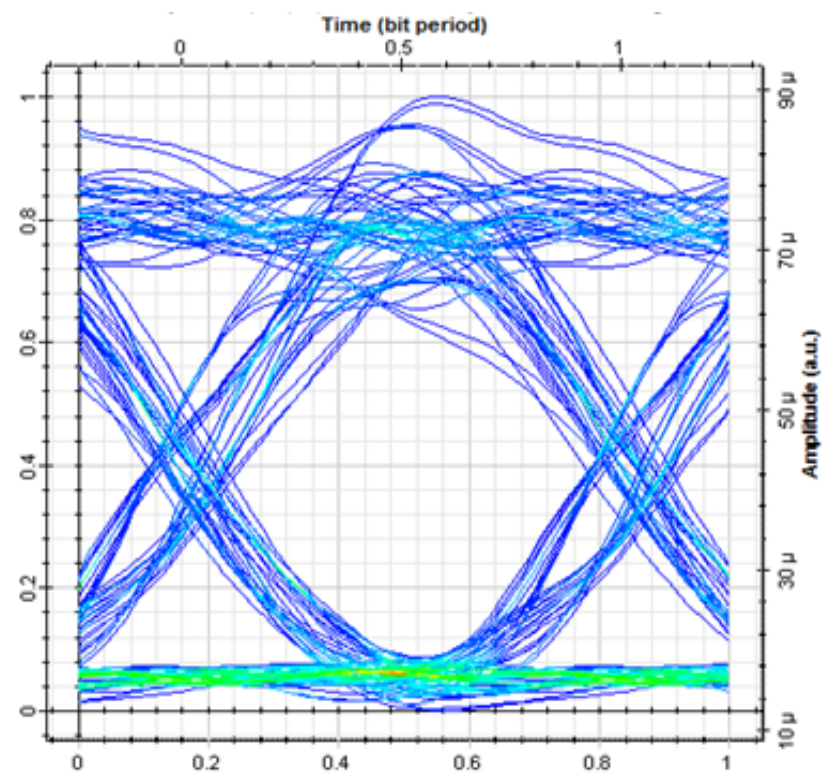

Figure 7. Eye diagram of the upstream signal at input power of $10 \mathrm{dBm}$. 
Table 2. Eye Diagram parameters comparison of the Uplink stage and downlink stage

\begin{tabular}{|c|c|c|}
\hline OQPSK Parameter & Uplink & Downlink \\
\hline Max Q. Factor & 6.98425 & 12.3429 \\
\hline Min BER & $1.18664 \mathrm{e}-012$ & $2.60574 \mathrm{e}-035$ \\
\hline Eye Height & $2.92781 \mathrm{e}-005$ & $4.97788 \mathrm{e}-005$ \\
\hline Threshold & $2.65933 \mathrm{e}-005$ & $1.62944 \mathrm{e}-006$ \\
\hline Decision Instant & 0.489474 & 0.536842 \\
\hline
\end{tabular}

The BER versus input optical power $P_{\text {in }}$ curves for the downlink and uplink are shown in Fig.8.It is noted from the figure that the BER for the uplink goes down slowly with increasing $P_{\text {in }}$ from $-2 \mathrm{dBm}$ to $10 \mathrm{dBm}$. The Min BER in the downlink is $2.6 \times 10^{-34}$ whereas the Min BER in the uplink is $1.2 \times 10^{-12}$.

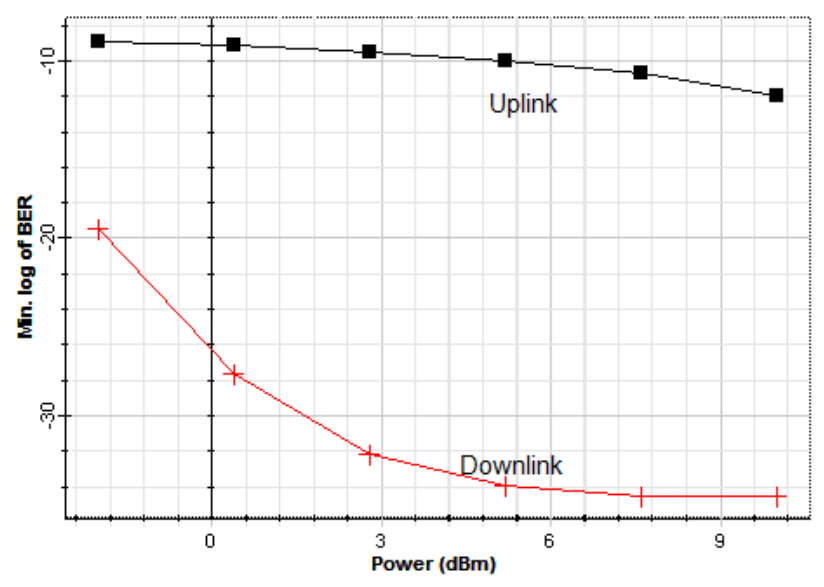

Figure 8. BER versus input power.

\subsection{Differential Phase Shift Keying (DPSK)}

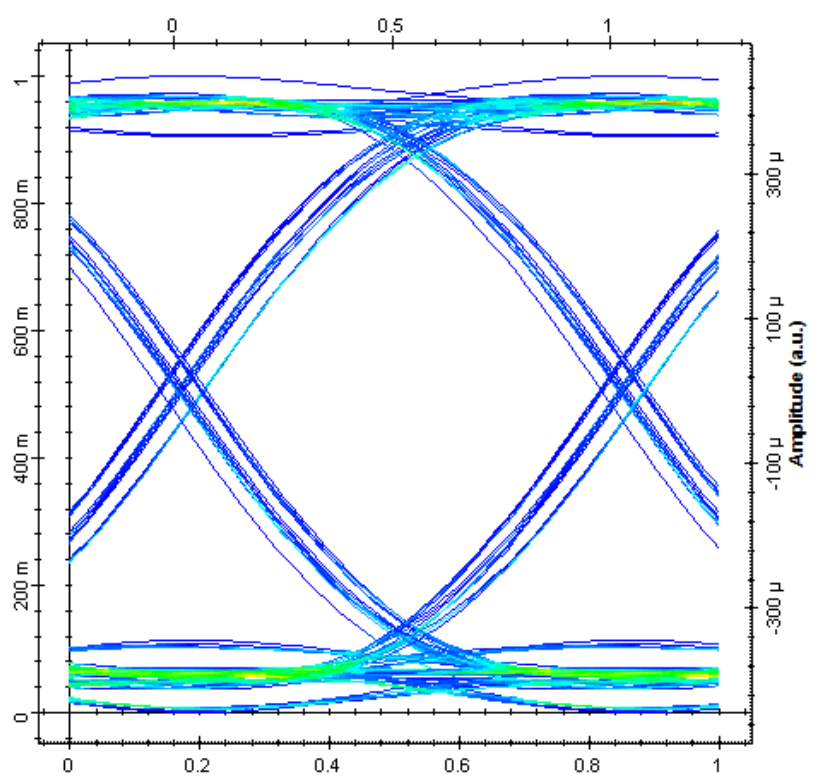

Figure 9. Eye Diagram of DPSK down link stage.
We have demonstrated a bidirectional ROF network based on reflective semiconductor optical amplifier (RSOA) utilizing a Differential Phase Shift keying modulation DPSK signal for down-link and Intensity Modulation (IM) for the upstream stage. We have used the same system modeled in Fig. 1 but we replace OQPSK modulator by DPSK modulator. Fig.9 demonstrates the Eye diagram for the DPSK downlink stage; it has a clear open eye pattern, an open eye pattern corresponds to minimal signal distortion. The eye diagram for the uplink stage is illustrated in Fig.10; we can see that the eye is less open compared with eye pattern illustrated in Fig.9. However it is a clear (not complete) eye pattern,it includes amplitude (noise) and phase (timing) errors.

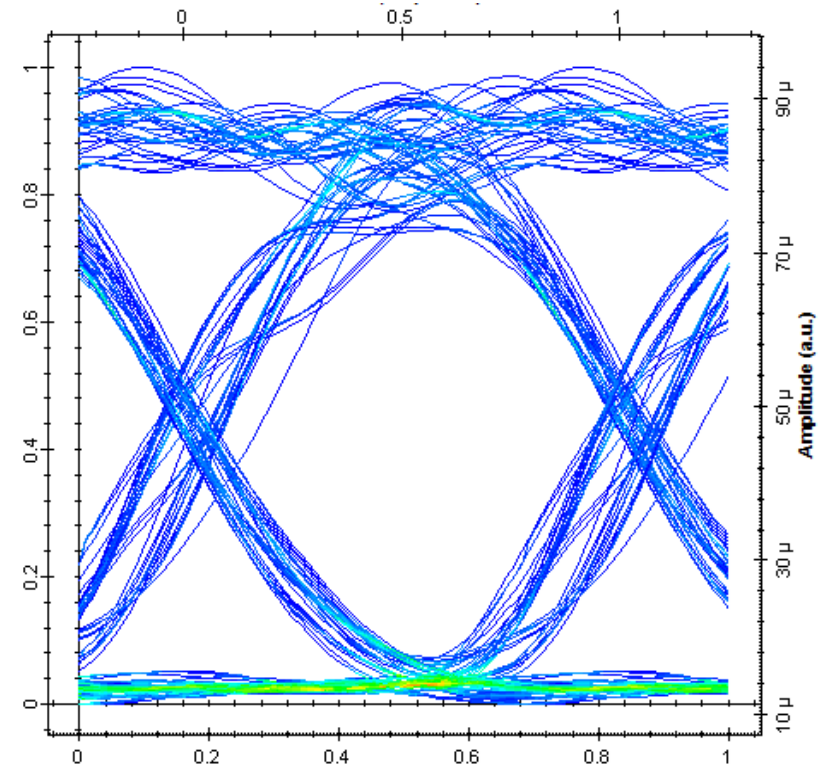

Figure 10. Eye Diagram of DPSK uplink stage.

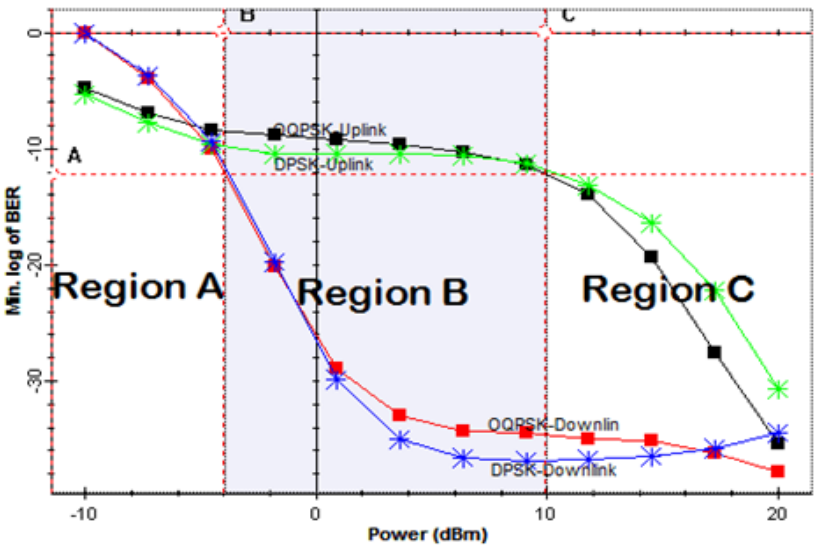

Figure 11. BER curves of OQPSK and DPSK

Now, we will make a detailed comparison between DPSK and an OQPSK. Fig.11 displays the measured downstream and upstream BER curves for both DPSK modulation and OQPSK modulation techniques. In general; a minimum acceptable BER rate is about $10^{-12}$. Referring to the figure; it is clear that system performance is affected by the power variation; the two systems perform well in region $B$ for the 
downlink stage and region $\mathrm{C}$ for uplink stage. In the uplink, OQPSK performs better than DPSK since its BER curves go down the BER curves of the DPSK.In the downlink, two systems have nearly the same BER curves form $-4 \mathrm{dBm}$ to 0 $\mathrm{dBm}$, for input power of more than 0dBm; BER curves of DPSK go down those of OQPSK and DPSK system performs in a better way.

The RSOA gain is affected by the input power variation. Figure 12 shows the relationship between the input power and the RSOA gain for both OQPSK and DPSK, it is obvious that there is an inverse proportion where the gain decreases as input power increases. Also it is clear that the DPSK gives larger RSOA gain than OQPSK does for the same input power [10]. Analysis shows that differential encoding approximately doubles the error rate compared to ordinary M-PSK but this may be overcome by only a small increase in input power. Furthermore, there will also be a physical channel between the transmitter and receiver in the communication system. This channel, in general, will introduce an unknown phase-shift to the PSK signal; in these cases the differential schemes can yield a better error-rate than the ordinary schemes which rely on precise phase information [11].

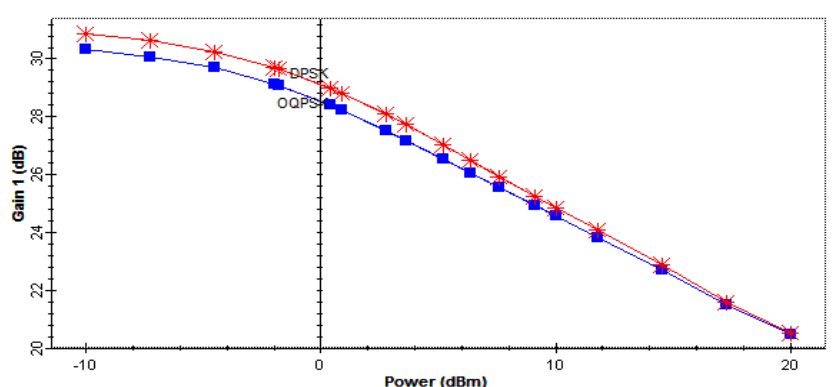

Figure 12. RSOA gain vs. input power for OQPSK and DPSK

\section{Conclusion}

This paper has introduced and compared two a novel bidirectional RoF systems, where an offset quadrature phase shift keying (OQPSK) or differential phase shift keying (DPSK) signals are used for down-link and an on-off keying (OOK) signal re-modulated for up-link. RSOA has been used for re-modulation of a down-link signal over $50 \mathrm{~km}$ SMF. The results showed that the OQPSK system performs better than DPSK in the uplink, while in the downlink the results were comparable. The results obtained here showed that both OQPSK and DPSK systems have potential applications in next-generation convergent wireless-wired optical network.

\section{REFERENCES}

[1] J. Part, C. Arellano and C. Bock, "Optical network unit based on a bidirectional reflective semiconductor optical amplifier for fiber-to-the-home networks," IEEE Photon, vol. 17, no. 1, pp. 250-252, Jan. 2005.

[2] G. C. Kassar, N.Calabretta, and I. T. Monroy, "Simultaneous optical carrier and radio frequency re-modulation in radio-over-fiber systems employing reflective SOA modulators," in Lasers and Electro-Optics Society, Lyngby, Oct. 2007, pp. 798-799.

[3] Gerd Keiser, Optical Fiber Communication, 3rd ed., International Editions, Ed.: McGraw Hill , 2000.

[4] B. Carbon, V. Girod and G. Maury. , "Optical Generation Of Microwave Functions," in Microwave Photonics for Emission and Detection of Broadband Communication Signals, Belgium, 2001.

[5] M. Webster, G. Wimpenny, K. Beacham and L. Crawford D. Wake, "Radio over fiber for mobile communications," IEEE, vol. 4, no. 2, April 2004.

[6] Simon Haykin, Digital Communications., Third Edition ed. Toronto, Canada: John Wiley \& Sons, 1988.

[7] Xianbin Yu, Timothy Braidwood Gibbon, and Idelfonso Tafur Monroy, "Bidirectional Radio-Over-Fiber System With Phase-Modulation Downlink and RF Oscillator-Free Uplink Using a Reflective SOA," IEEE, vol. 20, no. 24, pp. 2180-2182182, Dec. 2008

[8] Fady El-Nahal, "Bidirectional WDM-Radio over Fiber System with Sub Carrier Multiplexing Using a Reflective SOA and Cyclic AWGs," (IJACSA) International Journal of Advanced Computer Science and Applications, vol. 2, no. 8, pp. 93-96, August 2011.

[9] John G. Proakis, Digital Communications, Third Edtion ed. Singapore: McGraw Hill, 1995.

[10] Couch, Leon W. , Digital and Analog Communications, 6th ed. Upper Saddle River, USA: Prentice-Hall, 1997.

[11] Minhong Zhou, "Novel Modulation Technique for Radio-over-Fiber Systems," Concordia University, Canda, Thesis 978-0-494-40900-8, 2007. 